\title{
Pion and kaon distribution amplitudes up to twist-3 in the QCD instanton vacuum
}

\author{
Arthur Kock $\odot^{*}$ and Ismail Zahed ${ }^{\dagger}$ \\ Center for Nuclear Theory, Department of Physics and Astronomy, Stony Brook University, \\ Stony Brook, New York 11794-3800, USA
}

(Received 21 October 2021; accepted 2 November 2021; published 29 December 2021)

\begin{abstract}
We discuss the pion and kaon distribution amplitudes up to twist-3 in the context of the random instanton vacuum (RIV). We construct explicitly the pertinent quasipion and quasikaon distributions in the RIV, and analyze them in leading order in the diluteness factor, at a resolution fixed by the inverse instanton size. The distribution amplitudes (DA) follow from the large momentum limit. The results at higher resolution are discussed using QCD evolution, and compared to their asymptotic limits and some lattice results.
\end{abstract}

DOI: 10.1103/PhysRevD.104.116028

\section{INTRODUCTION}

Light cone distributions are central to the description of hard inclusive and exclusive processes. Thanks to factorization, a hard process factors into a perturbatively calculable contribution times pertinent parton distribution and fragmentation functions. Standard examples can be found in deep inelastic scattering, Drell-Yan process and jet production to cite a few.

The parton distribution functions are defined on the light front, and their moments usually fitted using large empirical data banks. They are not readily amenable to a nonperturbative and first principle formulation using lattice simulations. This situation has by now changed. Ji [1] has put forth the concept of spacelike quasiparton distributions that are perturbatively matched to the timelike light cone distributions [2-7]. This conjecture can be checked to hold non-perturbatively in two-dimensional QCD at next-toleading order in the large $N_{c}$ limit [8]. The quasiparton distribution matrix elements calculated in a fixed size Euclidean lattice QCD, have been argued to match those obtained through LSZ reduction in continuum Minkowski QCD, to all orders in perturbation theory [9]. Some variants of this formulation can be found in the form of pseudodistributions [10], and lattice cross sections [11]. A number of QCD lattice collaborations have implemented some of these ideas, with some reasonable success in extracting the light cone parton distributions.

\footnotetext{
*arthur.kock@stonybrook.edu

ismail.zahed@stonybrook.edu
}

Published by the American Physical Society under the terms of the Creative Commons Attribution 4.0 International license. Further distribution of this work must maintain attribution to the author(s) and the published article's title, journal citation, and DOI. Funded by SCOAP ${ }^{3}$.
A good understanding of the nonperturbative gauge fields responsible for chiral symmetry breaking was achieved in the context of the QCD instanton vacuum. Several QCD lattice simulations have shown that the bulk characteristics and correlations in the QCD vacuum are mostly unaffected by lattice cooling [12] where quantum effects are pruned, suggesting that semiclassical gauge and fermionic fields dominate the ground state structure. At weak coupling, instantons and anti-instantons are exact semiclassical gauge tunneling configurations with large actions and finite topological charge which support exact quark zero modes with specific chirality. They are at the origin of the spontaneous breaking of chiral symmetry and the emergence of a hadronic mass for the low-lying hadronic excitations such as the pion, kaon and nucleon. Orbitally excited hadrons are more sensitive to confinement, perhaps in the extended QCD instanton-dyon vacuum $[13,14]$, or in the QCD instanton vacuum with long P-vortices $[15,16]$.

In this work we follow up on our recent study of the quasidistributions in the random QCD instanton vacuum (RIV) [17]. More specifically, we will analyze the twoparticle pion and kaon quasidistributions up to twist-3 in the RIV, and extract the light cone distribution amplitudes in the large momentum limit. The moments of the twist-3 pion distribution amplitudes in an effective model of the RIV, and the twist-3 pion distribution amplitudes in a light front quark model using light cone signature, were recently discussed in $[18,19]$. Since the RIV vacuum is Euclidean, the distribution amplitudes are naturally extracted from the quasidistributions with space-like signature.

The outline of the paper is as follows: In Sec. II we briefly review the salient features of the RIV. In Sec. III we discuss the general structure of the pion and kaon in terms of the twist- 2 and twist- 3 contributions. Although the latter are subleading at asymptotic momenta in say the pion 
electromagnetic form factor, they still contribute substantially in the pre-asymptotic regime. In Sec. IV we define the quasipion and quasikaon distribution amplitudes and analyze them in the RIV using the power counting in the diluteness factor detailed in [17]. The massless and massive pseudoscalar and pseudotensor twist-3 pion and kaon distribution amplitudes are then extracted in the large momentum limit at the resolution fixed by the instanton size. The twist-2,-3 pion and kaon distribution amplitudes at higher resolution are discussed in Sec. V using the ERBL evolution and compared to their asymptotic limits and some lattice results. Our conclusions are in Sec. VI. Some useful details are found in the Appendices.

\section{INSTANTON EFFECTS}

The cooled QCD vacuum is populated with strong and inhomogeneous topological gauge configurations, i.e., instantons and anti-instantons as illustrated in Fig. 1 The bulk characteristics of this vacuum were predicted long ago [20]

$$
n_{I+\bar{I}} \approx 1 \mathrm{fm}^{-4}, \quad \rho \sim \frac{1}{3} \mathrm{fm} \sim \frac{1}{0.6} \mathrm{GeV}^{-1}
$$

for the instanton plus anti-instanton density and size, respectively. They combine in the dimensionless parameter

$$
\kappa \equiv \pi^{2} \rho^{4} n_{I+\bar{I}} \approx 3.186 \times 10^{-3}
$$

a measure of the diluteness of the instanton-anti-instanton ensemble in the QCD vacuum. Previous lattice simulations using cooling methods support these observations-see [21] for a review.

Instanton fields are strong, since their field strengths are large. For the dominant size instantons with $\rho \approx 0.30 \mathrm{fm}$ typical for chiral symmetry breaking, the fields are very strong at the center

$$
\sqrt{G_{\mu \nu}^{2}(0)}=\sqrt{192} / \rho^{2} \approx 5 \mathrm{GeV}^{2}
$$

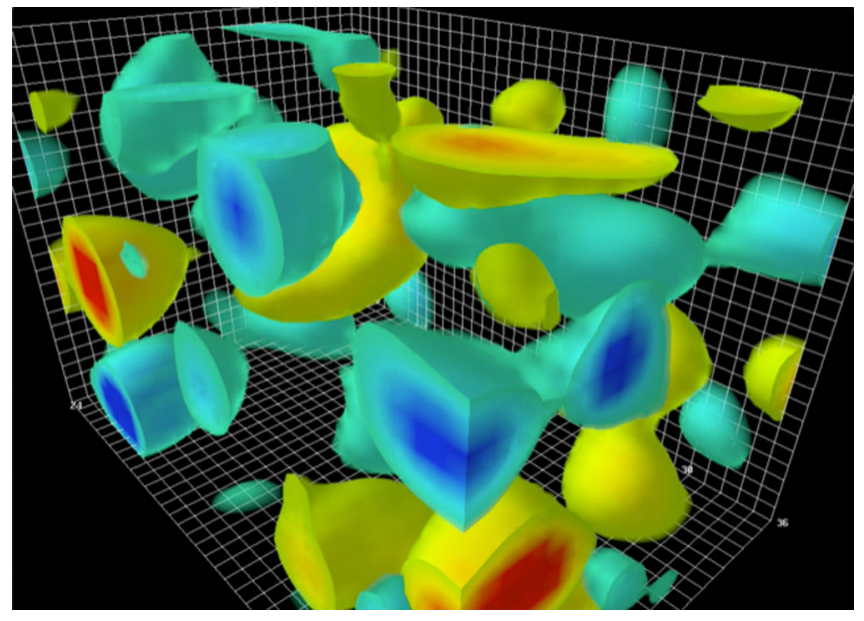

FIG. 1. Instantons (yellow) and anti-instantons (blue) configurations in the cooled YM vacuum [22].

Their scale is comparable to the matching scale in the hard and perturbative matching kernels [23] which may suggest nonperturbative improvements [24]. Their contribution can be assessed using semiclassics. The size distribution of the instantons and anti-instantons in the QCD vacuum is well captured semiempirically by $[25,26]$

$$
d n(\rho) \sim \frac{d \rho}{\rho^{5}}\left(\rho \Lambda_{\mathrm{QCD}}\right)^{b_{\mathrm{QCD}}} e^{-\alpha^{\prime} \rho^{2}}
$$

with $b_{\mathrm{QCD}}=11 N_{c} / 3-2 N_{f} / 3 \approx 9$ (one loop) and $\alpha^{\prime}=$ $1 / 2 m_{\rho}^{2}$ (rho meson slope).

\section{TWIST AND CHIRAL STRUCTURES OF THE DA OF THE PION}

In the QCD instanton vacuum, the pion DA is captured by the vertex $\pi^{-}(p) \rightarrow d_{i f \alpha}(k) u_{j g \beta}^{\dagger}(k-p)$, which corresponds formally to the connected amplitude

$$
\int_{-\infty}^{+\infty} \frac{p^{+} d z^{-}}{2 \pi} e^{-i x p \cdot z}\left\langle 0\left|\bar{u}_{\beta}(0)[0, z] d_{\alpha}(z)\right| \pi^{-}(p)\right\rangle=\left(+\frac{i f_{\pi}}{4} \gamma^{5}\left(\not p \phi_{\pi^{-}}^{A}(x)-\chi_{\pi} \phi_{\pi^{-}}^{P}(x)+i \chi_{\pi} \sigma_{\mu \nu} \frac{p^{\mu} p^{\prime \nu}}{p \cdot p^{\prime}} \frac{\phi_{\pi^{-}}^{\prime T}(x)}{6}\right)\right)_{\alpha \beta}
$$

and its conjugate

$$
\int_{-\infty}^{+\infty} \frac{p^{-} d z^{+}}{2 \pi} e^{i x p^{\prime} \cdot z}\left\langle\pi^{-}\left(p^{\prime}\right)\left|\bar{d}_{\beta}(z)[z, 0] u_{\alpha}(0)\right| 0\right\rangle=\left(-\frac{i f_{\pi}}{4} \gamma^{5}\left(p^{\prime} \phi_{\pi^{-}}^{A}(x)+\chi_{\pi} \phi_{\pi^{-}}^{P}(x)-i \chi_{\pi} \sigma_{\mu \nu} \frac{p^{\mu} p^{\prime \nu}}{p \cdot p^{\prime}} \frac{\phi_{\pi^{-}}^{\prime T}(x)}{6}\right)\right)_{\alpha \beta}
$$

up to twist-3. $[x, y]$ refers to the gauge link, $\sigma_{\mu \nu}=\frac{i}{2}\left[\gamma_{\mu}, \gamma_{\nu}\right]$, $\{\alpha, \beta\}$ represent spinor indices, and $\phi_{\pi}^{T \prime}(x)=\partial_{x} \phi_{\pi}^{T}(x)$. (3)-(4) are explicitly odd under $\mathrm{P}$ parity. Note that the 4 -vector $p_{\mu}^{\prime}$ appears in the DA of a pion with 4-vector $p_{\mu}$, in reference to the conjugate light-cone direction, with generally no relation to the second pion. In the DA of a pion with momentum $p_{\mu}^{\prime}$, the exchange $p \leftrightarrow p^{\prime}$ needs to be enforced, effectively flipping the sign of the last term. 
In (3), (4), and subsequent derivations, the ket $\left|\pi^{-}(P)\right\rangle$ refers to the physical negative-pion state. (Note the switch in flavors if the current $J_{\pi^{-}}(x)=\bar{d}(x) i \gamma^{5} u(x)$ is used to define the pion state). (3)-(4) can be inverted, to recast the pion twist-2 and twist-3 light-cone wave functions in explicit form

$$
\begin{aligned}
\phi_{\pi^{-}}^{A}(x) & =\frac{1}{i f_{\pi}} \int_{-\infty}^{+\infty} \frac{d z^{-}}{2 \pi} e^{i x p \cdot z}\left\langle 0\left|\bar{u}(0) \gamma^{+} \gamma_{5}[0, z] d(z)\right| \pi^{-}(p)\right\rangle \\
\phi_{\pi^{-}}^{P}(x) & =\frac{p^{+}}{f_{\pi} \chi_{\pi}} \int_{-\infty}^{+\infty} \frac{d z^{-}}{2 \pi} e^{i x p \cdot z}\left\langle 0\left|\bar{u}(0) i \gamma_{5}[0, z] d(z)\right| \pi^{-}(p)\right\rangle \\
\phi_{\pi^{-}}^{T \prime}(x) & =\frac{6}{f_{\pi} \chi_{\pi}} \frac{p^{\mu} p^{\prime \nu} p^{+}}{p \cdot p^{\prime}} \int_{-\infty}^{+\infty} \frac{d z^{-}}{2 \pi} e^{i x p \cdot z}\left\langle 0\left|\bar{u}(0) \sigma_{\mu \nu} \gamma_{5}[0, z] d(z)\right| \pi^{-}(p)\right\rangle
\end{aligned}
$$

with all DAs normalized to 1 . The prime in the last relation refers to $\partial_{x} \phi_{\pi^{-}}^{T}(x)$. The leading twist-2 DA $\phi_{\pi}^{A}(x)$ is chirallydiagonal. Its normalization to 1 is fixed by the weak pion decay constant $f_{\pi} \approx 130 \mathrm{MeV}$,

$$
\left\langle 0\left|\bar{u}(0) \gamma^{\mu}\left(1-\gamma^{5}\right) d(0)\right| \pi^{-}(p)\right\rangle=-\operatorname{Tr}\left(\gamma^{\mu}\left(1-\gamma^{5}\right)\left(\frac{i f_{\pi}}{4} \gamma^{5} \not p\right)\right) \int_{0}^{1} d x \phi_{\pi^{-}}^{A}(x) \equiv i f_{\pi} p^{\mu}
$$

Isospin symmetry and charge conjugation force $\phi_{\pi}(x)=\phi_{\pi}(\bar{x})$. The two twist-3 independent DAs $\phi_{\pi}^{P}(x)$ and $\phi_{\pi}^{T}(x)$ are chirally nondiagonal [27]. They are tied by the current identity

$$
\partial^{\nu}\left(\bar{u}(0) \sigma_{\mu \nu} \gamma_{5} d(z)\right)=-\partial_{\mu}\left(\bar{u}(0) i \gamma_{5} d(z)\right)+m \bar{u}(0) \gamma_{\mu} \gamma_{5} d(z)
$$

and share the same couplings. The value of the dimensionful coupling constant $\chi_{\pi}$ can be fixed by the divergence of the axial-vector current and the PCAC relation

$$
\left(m_{u}+m_{d}\right)\left\langle 0\left|\bar{u}(0) i \gamma^{5} d(0)\right| \pi^{-}(p)\right\rangle=-\left(m_{u}+m_{d}\right) \operatorname{Tr}\left(i \gamma^{5}\left(\frac{i f_{\pi}}{4} \gamma^{5} \chi_{\pi}\right)\right) \int_{0}^{1} d x \phi_{\pi}^{P}(x)=\left(m_{u}+m_{d}\right) f_{\pi} \chi_{\pi}
$$

with $\phi_{\pi}^{P}(x)$ normalized to 1 . Using the Gell-Mann-Oakes-Renner relation

$$
f_{\pi}^{2} m_{\pi}^{2}=-2\left(m_{u}+m_{d}\right)\langle\bar{q} q\rangle
$$

with $|\langle\bar{q} q\rangle| \approx(240 \mathrm{MeV})^{3}$, yield

$$
\chi_{\pi}=\frac{m_{\pi}^{2}}{\left(m_{u}+m_{d}\right)}
$$

The values of the quark masses depend on the renormalization scale $\mu$. Lattice simulations with fine lattices use $\mu \approx 2 \mathrm{GeV}$. However, for the DAs it is more appropriate to use a softer $\mu \approx 1 / \rho$ renormalization with slightly larger current quark masses giving $\chi_{\pi} \approx 1.2 \mathrm{GeV}$.

The twist-3 pion DAs asymptote $\phi_{\pi^{-}}^{P}(x) \rightarrow 1$ and $\phi_{\pi^{-}}^{T}(x) \rightarrow 6 x \bar{x}$ owing to their conformal collinear spin, with $\phi_{\pi^{-}}^{T \prime}(x) \rightarrow 6(\bar{x}-x)$. At large $Q^{2}$ their contribution is subleading in the pion electromagnetic form factor [28]

$$
\frac{f_{\pi}^{2} \chi_{\pi}^{2}}{Q^{4}} \int d x_{1} d x_{2} \frac{1}{\bar{x}_{1} \bar{x}_{2}}\left[\left(\frac{1}{\bar{x}_{2}}-1\right)+\left(\bar{x}_{2}-x_{2}\right)\left(\frac{1}{\bar{x}_{2}}+1\right)=2 \bar{x}_{2}\right]=2 \frac{f_{\pi}^{2} \chi_{\pi}^{2}}{Q^{4}} \int \frac{d x_{1}}{\bar{x}_{1}}
$$

\section{TWIST-3 QDA OF THE PION AND KAON}

The quasipion distribution distribution amplitudes (qPDA) variants of (5) are

$$
\tilde{\phi}_{\pi^{-}}^{A}\left(x, P_{z}\right)=\frac{i}{f_{\pi}} \int_{-\infty}^{\infty} \frac{d z}{2 \pi} e^{i \frac{x-\bar{x}}{2} P_{z} z}\left\langle 0\left|\bar{u}\left(z_{-}\right) \gamma^{z} \gamma^{5}\left[z_{-}, z_{+}\right] d\left(z_{+}\right)\right| \pi^{-}(p)\right\rangle
$$




$$
\begin{gathered}
\tilde{\phi}_{\pi^{-}}^{P}\left(x, P_{z}\right)=\frac{i P_{z}}{f_{\pi} \chi_{\pi}} \int_{-\infty}^{\infty} \frac{d z}{2 \pi} e^{i \frac{x-\bar{x}}{2} P_{z} z}\left\langle 0\left|\bar{u}\left(z_{-}\right) \gamma^{5}\left[z_{-}, z_{+}\right] d\left(z_{+}\right)\right| \pi^{-}(p)\right\rangle \\
\tilde{\phi}_{\pi^{-}}^{T \prime}\left(x, P_{z}\right)=\frac{6 P_{z}}{f_{\pi} \chi_{\pi}} \frac{P^{\mu} n^{\prime \nu}}{P \cdot n^{\prime}} \int_{-\infty}^{\infty} \frac{d z}{2 \pi} e^{i \frac{x-\bar{x}}{2} P_{z} z}\left\langle 0\left|\bar{u}\left(z_{-}\right) \sigma_{\mu \nu} \gamma^{5}\left[z_{-}, z_{+}\right] d\left(z_{+}\right)\right| \pi^{-}(p)\right\rangle
\end{gathered}
$$

where $z$ is a spacelike separation, $z_{ \pm}= \pm z / 2$, and $n^{\mu}=\{0,0,0,1\}$ is the unit-vector along the linear quark-separation (spacelike, $z$-direction here). For the corresponding $K^{-}$qPDAs, one would simply replace the $d$-quark with the $s$-quark, and switch to the state $\left|K^{-}(P)\right\rangle$. For finite $P_{z}$, the qPDAs can be matched with the corresponding light-cone DA counterparts (5) by an integration kernel, calculable order-by-order in powers of $\frac{\mu}{P_{z}}$ where $\mu$ represents any other mass-scale present $[1,23]$. However we will be taking the limit $P_{z} \rightarrow \infty$, where the matching becomes trivial $\tilde{\phi}\left(x, P_{z} \rightarrow \infty\right) \rightarrow \phi(x)$. Modulo $x$-independent prefactors, the twist-3 distributions only differ from the twist-2 distribution in their Dirac structure. We write this common factor as:

$$
\int_{-\infty}^{\infty} \frac{d z}{2 \pi} e^{i \frac{x-\bar{x}}{2} P_{z} z}\left\langle 0\left|\bar{u}\left(z_{-}\right) \Gamma\left[z_{-}, z_{+}\right] d\left(z_{+}\right)\right| \pi^{-}(p)\right\rangle
$$

Following the prescription of the present authors' previous paper [17], we insert the physical pion source and resum planar diagrams to leading order in the diluteness factor $\alpha \sim \sqrt{\kappa}$ to get

$-\frac{P^{2}+m_{\pi}^{2}}{P_{z} g_{\pi}} \int \frac{d^{4} k}{(2 \pi)^{4}} \delta\left(x-\frac{1}{2}-\frac{k_{z}}{P_{z}}\right) \operatorname{Tr}\left[\Gamma S_{1} O_{5}\left(P, p_{1}\right) S_{2}\right]$

where in going from (13) to (14), $S_{1}$ refers to the $d$-quark (or $s$-quark for the negative kaon) and $S_{2}$ refers to the $u$-quark. We have subsumed notation for the pion's onshell condition $\lim P^{2} \rightarrow-m_{\pi}^{2}$. The trace is over all indices, and $p_{1,2}^{\mu}=k^{\mu} \pm P^{\mu} / 2$ is the momentum carried by

(a)



each quark flavor. The resummed quark propagator $S_{1,2} \equiv S\left(p_{1,2}, m_{1,2}\right)$ is

$$
\begin{aligned}
S(k, m) & =\left(\frac{1}{\not k-i \sigma(k, m)}\right) \\
& \approx \frac{1}{k^{2}+M^{2}(0, m)}(\not k+i M(k, m))
\end{aligned}
$$

where $m$ is the current mass of the individual quark. The effective mass at LO in $\alpha$ is given by $\sigma(k, m) \approx$ $M(k, m)+\mathcal{O}\left(\alpha^{2}\right)$

$$
\begin{aligned}
M(k, m) & =\frac{M(k)}{\left(1+\xi^{2}\right)^{1 / 2}+\xi}+m \\
M(k) & =M(0)\left(\left|z\left(I_{0} K_{0}-I_{1} K_{1}\right)^{\prime}\right|^{2}\right)_{z=\frac{k \rho}{2}} \\
\xi & =\frac{m M(0) \rho^{2}}{8 \pi^{2} \kappa}
\end{aligned}
$$

with $M(k) \equiv M(k, 0)$ throughout. In the above approximation we have dropped the term $\Delta \sigma^{2} \equiv \sigma^{2}(k)-\sigma^{2}(0)$ because it only provides a correction to our final integrals which is subleading in $\alpha$. The benefit of this approximation is that our integrals will have vastly simplified $k^{\mu}$ dependence. In Fig. 2 we show the induced constituent quark mass (16) for the parameters of the instanton vacuum. In Fig. 2(a) we show $M(p, 0)$ solid-red curve versus $p$ in $\mathrm{GeV}$ units. The spread corresponds to $M(0)=383 \pm 39 \mathrm{MeV}$ and $\rho=0.313 \pm 0.016 \mathrm{fm}$. The open-circles are lattice generated quark masses in Coulomb gauge [29]. In Fig. 2(b) we show the dependence of the ratio $M(0, m) / M(0,0)$ on the current mass by the solid-blue curve for fixed $\xi$, and by the dashed-red curve for $\xi \ll 1$.

(b)



FIG. 2. (a) Effective quark mass $M(p)=M(p, 0)$, both axes $\mathrm{GeV}$; (b) Effective quark mass ratio, as a function of current quark mass. See text. 
The resummed pseudoscalar pion vertex is

$$
\begin{array}{r}
O_{5}\left(P, p_{1}\right) \approx \gamma^{5}\left(1+F_{5}\left(P, p_{1}\right)\right)+\alpha \bar{F}_{5}\left(P, p_{1}\right)+\mathcal{O}\left(\alpha^{2}\right) \\
F_{5}\left(P, p_{1}\right) \stackrel{P^{2} m_{\pi}^{2}}{\approx} \frac{g_{\pi}}{f_{\pi}} \sqrt{M\left(p_{1}\right)} \frac{1}{P^{2}+m_{\pi}^{2}} \sqrt{M\left(p_{2}\right)}
\end{array}
$$

where $g_{\pi}$ is the pseudoscalar pion-quark-quark coupling. For an explicit calculation of $g_{\pi}$ in the RIV framework, see Sec. III. C in [17]. Expanding to first order in $\alpha$, [14] keeping in mind that $M(k)=\alpha \sigma_{0}(k)$, the common factor becomes

$$
\begin{aligned}
- & \frac{P^{2}+M_{\pi}^{2}}{P_{z} g_{\pi}} \int \frac{d^{4} k}{(2 \pi)^{4}} \delta\left(x-\frac{1}{2}-\frac{k_{z}}{P_{z}}\right)\left\{\operatorname{Tr}\left[\Gamma \frac{\not p_{1}}{p_{1}^{2}+M_{1}^{2}} \gamma^{5}\left(1+F_{5}\left(p_{1}, p_{2}\right)\right) \frac{\not p_{2}}{p_{2}^{2}+M_{2}^{2}}\right]\right. \\
& +\operatorname{Tr}\left[\Gamma\left(\frac{i \alpha \sigma_{0}\left(p_{1}\right)}{p_{1}^{2}+M_{1}^{2}}\right) \gamma^{5}\left(1+F_{5}\left(p_{1}, p_{2}\right)\right) \frac{\not p_{2}}{p_{2}^{2}+M_{2}^{2}}\right]+\operatorname{Tr}\left[\Gamma \frac{\not p_{1}}{p_{1}^{2}+M_{1}^{2}} \gamma^{5}\left(1+F_{5}\left(p_{1}, p_{2}\right)\right)\left(\frac{i \alpha \sigma_{0}\left(p_{2}\right)}{p_{2}^{2}+M_{2}^{2}}\right)\right] \\
& \left.+\operatorname{Tr}\left[\Gamma \frac{\not p_{1}}{p_{1}^{2}+M_{1}^{2}} \alpha \bar{F}_{5}\left(P, p_{1}\right) \frac{\not p_{2}}{p_{2}^{2}+M_{2}^{2}}\right]\right\}+\mathcal{O}\left(\alpha^{2}\right)
\end{aligned}
$$

with $M_{1,2} \equiv M\left(0, m_{1,2}\right)$ being the effective mass for each quark.

In (19) there are four traces: the first is of order $\alpha^{0}$, the next three are of order $\alpha^{1}$. If $\Gamma$ contains an odd number of $\gamma$ 's (e.g., $\Gamma=\gamma^{z} \gamma^{5}$ ), the first trace term at order $\alpha^{0}$ has vanishing Dirac trace, whereas the remaining three do not have vanishing Dirac trace. This is the case when calculating the twist-2 qPDA. However if $\Gamma$ contains an even number of $\gamma$ 's (e.g., $\Gamma=\gamma^{5}, \sigma_{\mu \nu} \gamma^{5}$ ), then the first term has nonvanishing Dirac trace. At next to leading order (NLO) in $\alpha$, the second and third terms vanish. The fourth term involving $\bar{F}_{5}$ does not have vanishing Dirac trace, and requires special attention. This is the case with the twist-3 distributions, which we are considering here. In our previous paper [17] we showed that this term vanishes for the axial-vector twist-2 DA, $\Gamma=\gamma^{z} \gamma^{5}$. We now make explicit the leading contributions in $\alpha \sim \sqrt{\kappa}$ to the twist-3 DAs, $\phi_{0}^{P}(x)$ and $\phi_{0}^{T}(x)$. We also recap the similar expression for the twist-2 DA, $\phi_{0}^{A}(x)$.

\section{A. Pseudoscalar, $\Gamma=\gamma^{5}$}

The calculation of $\tilde{\phi}_{0}^{P}(x$,$) begins by reinstating the$ appropriate prefactor in (19). Out of the first three zeromode contributions in (19), only the first has nonvanishing Dirac trace

$$
\begin{aligned}
\tilde{\phi}_{0}^{P}\left(x, P_{z}\right) & =\frac{i P_{z}}{f_{\pi} \chi_{\pi}} \frac{-P^{2}}{P_{z} g_{\pi}} \int \frac{d^{4} k}{(2 \pi)^{4}} \delta\left(x-\frac{1}{2}-\frac{k_{z}}{P_{z}}\right) \operatorname{Tr}\left[\gamma^{5} \frac{\not p_{1}}{p_{1}^{2}+M_{1}^{2}} \gamma^{5}\left(1+F_{5}\left(p_{1}, p_{2}\right)\right) \frac{\not p_{2}}{p_{2}^{2}+M_{2}^{2}}\right] \\
& =\frac{4 i N_{c}}{f_{\pi}^{2} \chi_{\pi}} \int \frac{d^{2} k_{\perp} d k_{4} d k_{z}}{(2 \pi)^{4}} \delta\left(x-\frac{1}{2}-\frac{k_{z}}{P_{z}}\right)\left(M\left(p_{1}\right) M\left(p_{2}\right)\right)^{1 / 2} \frac{p_{1} \cdot p_{2}}{\left(p_{1}^{2}+M_{1}^{2}\right)\left(p_{2}^{2}+M_{2}^{2}\right)} \\
& =\frac{4 i N_{c} P_{z}}{f_{\pi}^{2} \chi_{\pi}} \int \frac{d^{2} k_{\perp} d k_{4}}{(2 \pi)^{4}}\left(M\left(p_{1}\right) M\left(p_{2}\right)\right)^{1 / 2} \frac{p_{1} \cdot p_{2}}{\left(p_{1}^{2}+M_{1}^{2}\right)\left(p_{2}^{2}+M_{2}^{2}\right)}
\end{aligned}
$$

where the delta function has set $P_{1, z}=x P_{z}$ and $P_{2, z}=-\bar{x} P_{z}$. Since

$$
p_{1}-p_{2}=P \Rightarrow p_{1} \cdot p_{2}=\frac{1}{2}\left[p_{1}^{2}+p_{2}^{2}+m_{\pi}^{2}\right]
$$

the last term in (20) can be recast in the form

$$
\tilde{\phi}_{0}^{P}\left(x, P_{z}\right)=\frac{2 i N_{c} P_{z}}{f_{\pi}^{2} \chi_{\pi}} \int \frac{d^{2} k_{\perp} d k_{4}}{(2 \pi)^{4}}\left(M\left(p_{1}\right) M\left(p_{2}\right)\right)^{1 / 2} \frac{p_{1}^{2}+p_{2}^{2}+m_{\pi}^{2}}{\left(p_{1}^{2}+M_{1}^{2}\right)\left(p_{2}^{2}+M_{2}^{2}\right)}
$$

Notice that for the $k_{4}$-integration, the pole and branch point structure is exactly the same as in the twist- 2 case. Therefore we evaluate in the same way, by Wick-rotating and shifting in $k_{4}$, into the Minkowski domain as we detail in Appendix A. Taking the final limit $P_{z} \rightarrow \infty$, including the finite pion mass [17], and simplifying the pre-factor using $\chi_{\pi}=-2\langle\bar{\psi} \psi\rangle / f_{\pi}^{2}$, the final result for the leading-order pseudoscalar DA is

$$
\tilde{\phi}_{0}^{P}(x)=i \frac{N_{c}}{2\langle\bar{\psi} \psi\rangle} \frac{\theta(x \bar{x})}{x \bar{x}} \int \frac{d^{2} k_{\perp}}{(2 \pi)^{3}} M\left(\frac{k_{\perp}}{\lambda \sqrt{x \bar{x}}}\right) \frac{k_{\perp}^{2}+\bar{x}^{2} M_{1}^{2}+x^{2} M_{2}^{2}}{k_{\perp}^{2}+\bar{x} M_{1}^{2}+x M_{2}^{2}-x \bar{x} m_{\pi}^{2}}
$$


The requisite $x \leftrightarrow \bar{x}, m_{1} \leftrightarrow m_{2}$ symmetry is manifest. For plotting we use phenomenological values $M(0)=$ $383 \mathrm{MeV}, \quad|\langle\bar{\psi} \gamma \psi\rangle|=(240 \mathrm{MeV})^{3}$, and $\rho=0.313 \mathrm{fm}$. The normalizing values of $\lambda$ are provided in Table $\mathrm{I}$ in Appendix B. Unevolved plots of (23) are shown in Fig. 3 for both the limit of a small instanton size $\rho \rightarrow 0$ and typical instanton size $\rho=0.313 \mathrm{fm}$. For the pion, there is no perceptible difference in (23) between using physical masses $\left(m_{\pi}=140 \mathrm{MeV}, m_{u}=3 \mathrm{MeV}, m_{d}=7 \mathrm{MeV}\right)$ and the chiral counterparts $\left(m_{\pi, u, d}=0\right)$. For the kaon we use $m_{K}=494 \mathrm{MeV}, m_{u}=3 \mathrm{MeV}, m_{s}=136 \mathrm{MeV}$. The small-size instanton limit $(\rho \rightarrow 0)$ corresponds to very high resolution and is commensurate with the QCD asymptotic result as expected.

\section{B. Pseudotensor, $\Gamma=\sigma_{\mu \nu} \gamma^{5}$}

The only difference with the pseudoscalar case will be that instead of having a factor $i \operatorname{Tr}[\not \not \not p]$, we will have

$$
\begin{aligned}
6 \frac{P_{\mu} n_{\nu}}{P \cdot n} \operatorname{Tr}\left[\sigma^{\mu \nu} \not p 1 \not p 2\right] & =-24 i \frac{P_{\mu} n_{\nu}}{P \cdot n}\left(p_{1}^{\mu} p_{2}^{\nu}-p_{2}^{\mu} p_{1}^{\nu}\right) \\
& =\frac{-24 i}{P \cdot n}\left[\left(P \cdot p_{1}\right)\left(n \cdot p_{2}\right)-\left(n \cdot p_{1}\right)\left(P \cdot p_{2}\right)\right]
\end{aligned}
$$

where $n^{\mu}$ is the tangent vector to the spacelike quark separation line in (12), $n^{\mu}=\hat{z}^{\mu}$. Making this replacement in (20), we get

$$
\tilde{\phi}_{0}^{T \prime}\left(x, P_{z}\right)=\frac{-24 i N_{c}}{f_{\pi}^{2} \chi_{\pi}} \int \frac{d^{2} k_{\perp} d k_{4}}{(2 \pi)^{4}}\left(M\left(p_{1}\right) M\left(p_{2}\right)\right)^{1 / 2} \frac{\left(P \cdot p_{1}\right)\left(n \cdot p_{2}\right)-\left(n \cdot p_{1}\right)\left(P \cdot p_{2}\right)}{\left(p_{1}^{2}+M_{1}^{2}\right)\left(p_{2}^{2}+M_{2}^{2}\right)}
$$

As before, we Wick-rotate and shift the $k_{4}$ integration, $k_{4} \rightarrow i\left(k_{4}+\left(x-\frac{1}{2} E\right)\right)$, leaving us with

$$
\tilde{\phi}_{0}^{T \prime}\left(x, P_{z}\right)=\frac{24 N_{c}}{f_{\pi}^{2} \chi_{\pi}} \int \frac{d^{2} k_{\perp} d k_{4}}{(2 \pi)^{4}}\left(M\left(y_{1}\right) M\left(y_{2}\right)\right)^{1 / 2} \frac{\left(P \cdot y_{1}\right)\left(n \cdot y_{2}\right)-\left(n \cdot y_{1}\right)\left(P \cdot y_{2}\right)}{\left(y_{1}^{2}+M_{1}^{2}\right)\left(y_{2}^{2}+M_{2}^{2}\right)}
$$

To evaluate the integrand we use the same kinematics as in (A2). To perform the $k_{4}$ integral, we follow the same procedure as in the pseudoscalar case - use the modified effective mass, then integrate the remaining rational function using Cauchy's residue theorem. The final result for the integrated $\phi^{T}(x)$ is

$$
\tilde{\phi}_{0}^{T}(x)=\frac{-3 i N_{c}}{\langle\bar{\psi} \psi\rangle} \theta(x \bar{x}) \int_{0}^{x} d v \int \frac{d^{2} k_{\perp}}{(2 \pi)^{3}} M\left(\frac{k_{\perp}}{\lambda \sqrt{v \bar{v}}}\right) \frac{1}{v \bar{v}} \frac{(\bar{v}-v) k_{\perp}^{2}+\bar{v}^{2} M_{1}^{2}-v^{2} M_{2}^{2}}{k_{\perp}^{2}-v \bar{v} m_{\pi}^{2}+\bar{v} M_{1}^{2}+v M_{2}^{2}}
$$

Again, the requisite $x \leftrightarrow \bar{x}, m_{1} \leftrightarrow m_{2}$ symmetry is manifest (the integrand is odd under this transformation). Unevolved plots of (27) are shown in Fig. 3 for phenomenological and limiting values of $\rho$. In the case that $\rho \rightarrow 0$ (high resolution), the pseudotensor DA approaches its asymptotic form. Once again, the normalizing values of $\lambda$ are given in Table I in Appendix B.
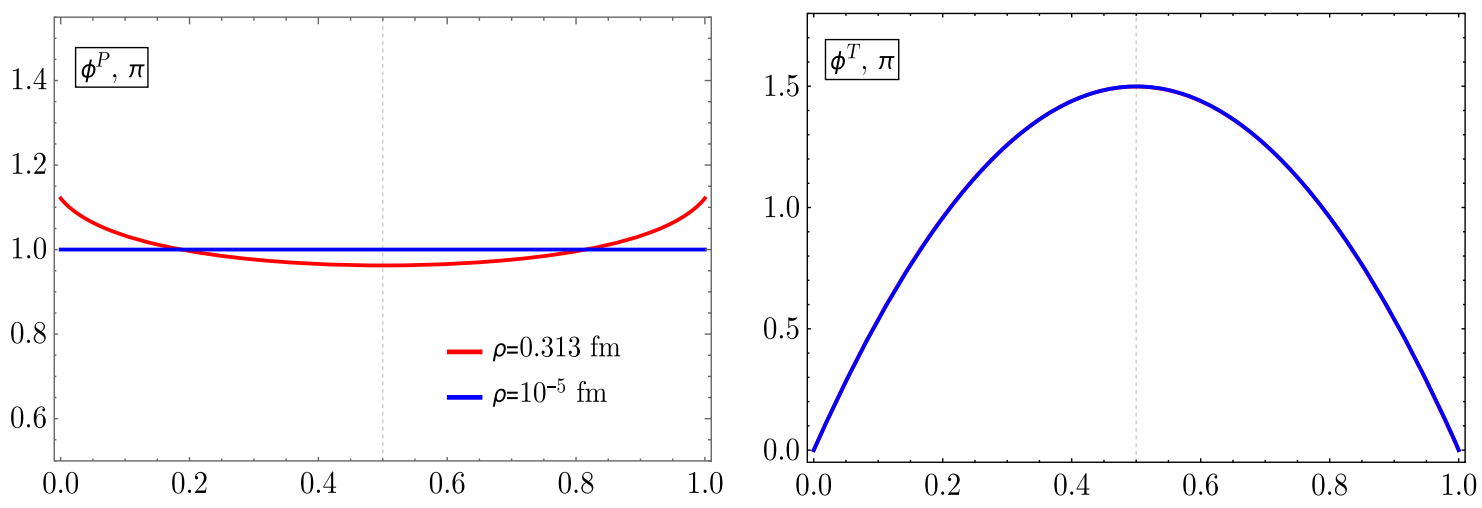

FIG. 3. Pseudoscalar and pseudotensor pion DAs at different instanton sizes or resolution. The $\rho \approx 0$ curves (solid blue) are indiscernible from the asymptotic forms. For the pseudotensor DA $\phi^{T}$, both for phenomenological and small $\rho$, the curves are indistinguishable from the asymptotic form $6 x \bar{x}$. 


\section{Axial-vector, twist-2, $\Gamma=\gamma^{z} \gamma^{5}$}

Here we generalize a key expression from our previous paper [17]: the twist-2 DA at leading order in $\alpha$, now including finite current quark masses $m_{1,2}$ for the pseudoscalar meson $P$. It is given by

$$
\begin{aligned}
\phi_{P}^{A}(x) \approx & \frac{2 N_{c}}{f_{P}^{2}} \theta(x \bar{x}) \int \frac{d^{2} k_{\perp}}{(2 \pi)^{3}} M\left(\tilde{k}_{\perp}\right) \\
& \times \frac{\bar{x} M\left(\tilde{k}_{\perp}, m_{1}\right)+x M\left(\tilde{k}_{\perp}, m_{2}\right)}{k_{\perp}^{2}-x \bar{x} m_{P}^{2}+\bar{x} M_{1}^{2}+x M_{2}^{2}}
\end{aligned}
$$

with $\tilde{k}_{\perp}=k_{\perp} / \lambda \sqrt{x \bar{x}}$. We use $f_{\pi}=130 \mathrm{MeV}$ for massive pions and $f_{K}=155 \mathrm{MeV}$ for massive kaons. Unevolved plots of (28) are shown in Fig. 4 for phenomenological and limiting values of $\rho$. For $\rho \rightarrow 0$ the curves tend toward a normalized step-function, rather than toward the asymptotic distribution $6 x \bar{x}$. This type of curve has been noted for chiral quark models with point interactions [30,31], and some bound-state resummations [32].

\section{QCD EVOLUTION}

The two-particle twist 2 and 3 DAs in the random instanton vacuum (RIV) are defined at a low renormalization scale set by the typical inverse instanton size $Q_{0}=1 / \rho=631 \mathrm{GeV}$. Assuming factorization, their forms at higher renormalization scales follow from QCD evolution equations

$$
\begin{aligned}
\phi^{A}(x, Q)= & 6 x \bar{x} \sum_{n=0}^{\infty} a_{n}\left(Q_{0}\right)\left(\frac{\alpha_{s}\left(Q^{2}\right)}{\alpha_{s}\left(Q_{0}^{2}\right)}\right)^{\gamma_{n}^{A} / \beta_{0}} \\
& \times C_{n}^{3 / 2}(x-\bar{x}) \\
\phi^{P}(x, Q)= & 1+\sum_{n=1}^{\infty} b_{n}\left(Q_{0}\right)\left(\frac{\alpha_{s}\left(Q^{2}\right)}{\alpha_{s}\left(Q_{0}^{2}\right)}\right)^{\gamma_{n}^{P} / \beta_{0}} \\
& \times C_{n}^{1 / 2}(x-\bar{x})
\end{aligned}
$$

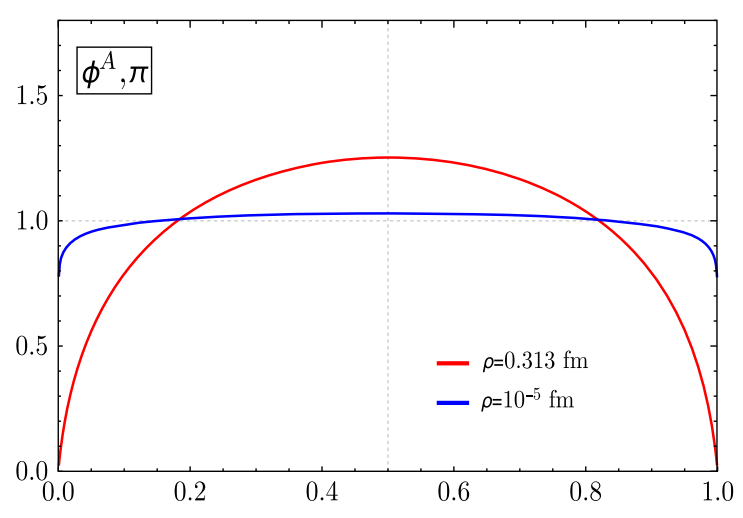

$\phi^{T}(x, Q)=6 x \bar{x}\left[1+\sum_{n=1}^{\infty} c_{n}\left(Q_{0}\right)\left(\frac{\alpha_{s}\left(Q^{2}\right)}{\alpha_{s}\left(Q_{0}^{2}\right)}\right)^{\gamma_{n}^{T} / \beta_{0}} C_{n}^{3 / 2}(x-\bar{x})\right]$

with the anomalous dimensions $\gamma_{n}^{A, P, T}$ given by [33]

$$
\begin{aligned}
& \gamma_{n}^{A}=C_{F}\left[-3+4 \sum_{j=1}^{n+1} \frac{1}{j}-\frac{2}{(n+1)(n+2)}\right] \\
& \gamma_{n}^{P}=C_{F}\left[-3+4 \sum_{j=1}^{n+1} \frac{1}{j}-\frac{8}{(n+1)(n+2)}\right] \\
& \gamma_{n}^{T}=C_{F}\left[-3+4 \sum_{j=1}^{n+1} \frac{1}{j}\right]
\end{aligned}
$$

Here $C_{n}^{m}(z)$ are Gegenbauer polynomials, $C_{F}=$ $\left(N_{c}^{2}-1\right) / 2 N_{c}$ is the quadratic-Casimir in the fundamental representation, $\alpha_{s}\left(Q^{2}\right)=4 \pi /\left(\beta_{0} \ln \left(Q^{2} / \Lambda^{2}\right)\right)$ is the oneloop running QCD coupling, $\beta_{0}=\frac{11}{3} N_{c}-\frac{2}{3} N_{f}$, and $\Lambda=250 \mathrm{MeV}$. One can easily verify that the normalizations are preserved under QCD evolution, as they should be. Owing to the orthogonality of the Gegenbauer polynomials, the initial coefficients are given by

$$
a_{n}\left(Q_{0}\right)=\frac{2(2 n+3)}{3(n+1)(n+2)} \int_{0}^{1} d y C_{n}^{3 / 2}(y-\bar{y}) \phi^{A}\left(y, Q_{0}\right)
$$

$$
\begin{aligned}
& b_{n}\left(Q_{0}\right)=(2 n+1) \int_{0}^{1} d y C_{n}^{1 / 2}(y-\bar{y}) \phi^{P}\left(y, Q_{0}\right) \\
& c_{n}\left(Q_{0}\right)=\frac{2(2 n+3)}{3(n+1)(n+2)} \int_{0}^{1} d y C_{n}^{3 / 2}(y-\bar{y}) \phi^{T}\left(y, Q_{0}\right)
\end{aligned}
$$

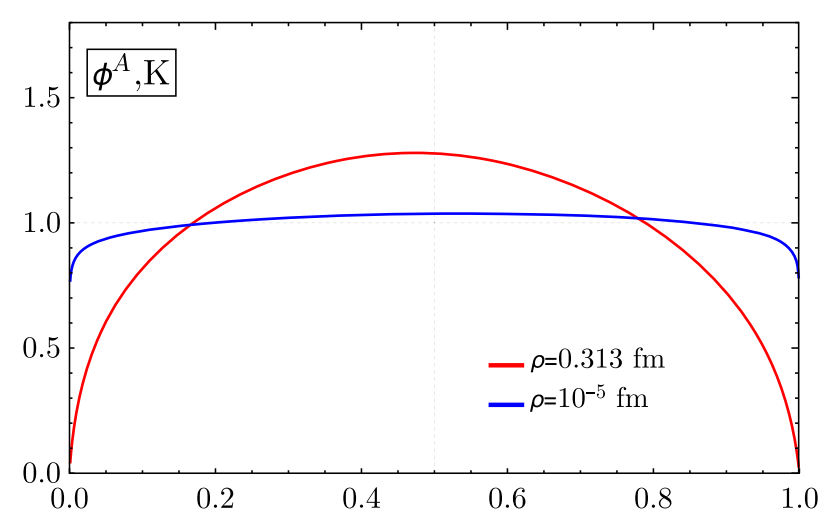

FIG. 4. Twist-2 (axial-vector) pion and kaon DAs at different instanton sizes or resolution. The normalizing values of $\lambda$ for the $\rho=10^{-5}$ fm curves are as follows: $\lambda_{\pi}=7.51, \lambda_{K}=9.7$ (for the phenomenological curves see Appendix B). For both the pion and kaon, the $\rho \approx 0$ curves (solid blue) tend toward a normalized step-function $\theta(x \bar{x})$. 
The twist-2 and twist-3 DAs, evolved to $Q=2 \mathrm{GeV}$, are shown in Fig. 5. All curves are shown at the same renormalization scale. Our twist-2 pion DA shows a shape slightly broader than the asymptotic form. This shape has been seen in recent lattice calculations [2,34]. In some light-front constituent quark models, a shape slightly narrower than the asymptotic form is seen-we do not display this curve in Fig. 5(a) because of a mismatch in renormalization schemes [35]. The empirical pion twist-2 DA extracted from dijet data by the E791 collaboration is in agreement with all curves shown Fig. 5(a), though the precise shape is obscured by uncertainties [36]. The same broad shape is seen in our twist2 kaon DA, Fig. 5(b), although we see a smaller asymmetry than other phenomenological approaches.
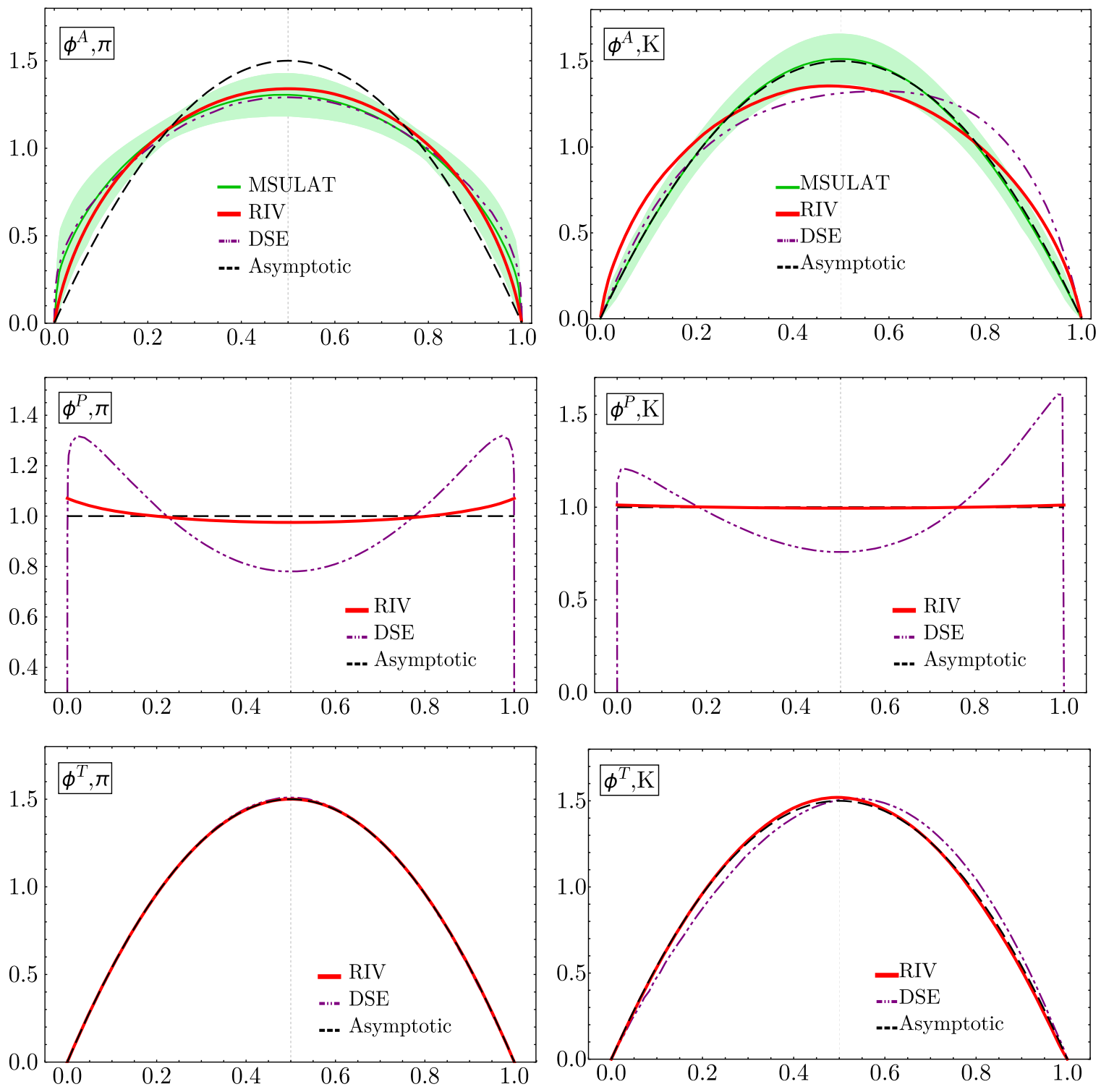

FIG. 5. ERBL evolution of DAs, compared with data. All curves are given at $Q=2 \mathrm{GeV}$. RIV (red-solid-thick) represents our current work, the random instanton vacuum approach, given by Eqs. (23), (27), and (28). MSULAT curves (green-solid-bands) are recent lattice computations utilizing the LaMET framework [34]. DSE curves (purple-dash-dot-dot) are from [37] which utilized Dyson-Schwinger equations with Bethe-Salpeter amplitudes. 


$$
\begin{aligned}
b_{1}^{\pi}\left(Q_{0}\right) & =8.4 \times 10^{-5} \quad b_{2}^{\pi}\left(Q_{0}\right)=1.74 \times 10^{-2} \\
\left|\frac{b_{1}^{\pi}\left(Q_{0}\right)}{b_{2}^{\pi}\left(Q_{0}\right)}\right| & =4.82 \times 10^{-3} \\
b_{1}^{K}\left(Q_{0}\right) & =1.41 \times 10^{-4} \quad b_{2}^{K}\left(Q_{0}\right)=3.46 \times 10^{-3} \\
\left|\frac{b_{1}^{K}\left(Q_{0}\right)}{b_{2}^{K}\left(Q_{0}\right)}\right| & =4.07 \times 10^{-2}
\end{aligned}
$$

\section{CONCLUSIONS}

Cooled lattice gauge configurations display strongly inhomogeneous instanton and anti-instanton configurations. The dilute QCD instanton vacuum in its simplified RIV form capture the essentials physics of these tunneling configurations at low resolution. Each tunneling traps a zero mode of a given chirality for each flavor, breaking dynamically chiral symmetry. The disordering of these zero modes leads to a multitude of multiquark condensates and a running constituent quark mass $[21,38]$.

In the RIV the pion quasi-DA is a state made of zeromodes and nonzero-modes that interact collectively. While still complex, this state can be organized in terms of the RIV diluteness factor $\alpha \sim \sqrt{\kappa}$. In leading order in $\alpha$, the twist-2 contribution to the pion quasi-DA involves both the zero-modes and nonzero-modes as we have analyzed in details in [17] and smoothly yields the pion DA in the large momentum limit. quasi-DA are dominated solely by the zero-modes owing to their pseudoscalar and pseudotensor content. They also yield smoothly the pion DA in the large momentum limit. In all cases the DA follows from the large momentum limit of the quasi-DA.

Our evolved results for the twist-2 (axial-vector) and twist-3 (tensor) pion and kaon DA amplitudes, are very close to the DSE results, and consistent with the recently reported lattice results for the twist-2. Our evolved results for the twist-3 (pseudoscalar) for the pion and kaon DA amplitudes are different from those obtained using the DSE, but very close to the QCD asymptotic results. It is rather remarkable, that our pion and kaon DA amplitudes probe specifically the running emergent topological quark mass in the dilute RIV, which is the dominant component of the QCD vacuum at low resolution.

\section{ACKNOWLEDGMENTS}

This work is supported by the Office of Science, U.S. Department of Energy under Contract No. DE-FG88ER40388.

\section{APPENDIX A: DETAILS OF EQ. (23)}

We start from (22) and perform the analytical continuation $k_{4} \rightarrow i k_{4}$, followed by the shift $k_{4} \rightarrow k_{4}+\left(x-\frac{1}{2}\right) E$. The result is

$$
\begin{aligned}
\tilde{\phi}_{0}^{P}\left(x, P_{z}\right)= & \frac{2 i N_{c} P_{z}}{f_{\pi}^{2} \chi_{\pi}} \int \frac{d^{2} k_{\perp}\left(i d k_{4}\right)}{(2 \pi)^{4}}\left(M\left(y_{1}\right) M\left(y_{2}\right)\right)^{1 / 2} \\
& \times \frac{y_{1}^{2}+y_{2}^{2}+m_{\pi}^{2}}{\left(y_{1}^{2}+M_{1}^{2}-i \epsilon\right)\left(y_{2}^{2}+M_{2}^{2}-i \epsilon\right)}
\end{aligned}
$$

where

$$
\begin{aligned}
y_{1}^{\mu} & =\left(\vec{k}_{\perp}, x P_{z}, i\left(k_{4}+x E\right)\right) \\
y_{1}^{2} & =-k_{4}\left(k_{4}+2 x E\right)+k_{\perp}^{2}-x^{2} m_{\pi}^{2} \\
y_{1}^{2}+M_{1}^{2}-i \epsilon & =-\left(k_{4}-k_{4+}\right)\left(k_{4}-k_{4-}\right) \\
y_{2}^{\mu} & =\left(\vec{k}_{\perp},-\bar{x} P_{z}, i\left(k_{4}-\bar{x} E\right)\right) \\
y_{2}^{2} & =-k_{4}\left(k_{4}-2 \bar{x} E\right)+k_{\perp}^{2}-\bar{x}^{2} m_{\pi}^{2} \\
y_{2}^{2}+M_{2}^{2}-i \epsilon & =-\left(k_{4}-\bar{k}_{4+}\right)\left(k_{4}-\bar{k}_{4-}\right) .
\end{aligned}
$$

The emergent constituent quark mass $M(y)$ is characterized by branch points. In [17] we have shown that the analysis retaining the branch points in $M(y)$ is similar to the one following from the modified effective mass at large $P_{z}$,

$$
M(y) \rightarrow M\left(\frac{k_{\perp}}{\lambda \sqrt{|x \bar{x}|}}\right)
$$

This removes the explicit $k_{4}$-dependence in $M(y)$, so we can evaluate the $k_{4}$ integral by residues. The value of $\lambda$ is then fixed to reproduce unit normalization of the DA. Note that in [17], we used the additional constraint $k_{\perp}>M(0)$ in the cutoff, which does not affect the power counting, but is not necessary.

With the above in mind, the integrand in (A1) has 4 poles in the complex $k_{4}$ plane, $\left\{k_{4 \pm}, \bar{k}_{ \pm}\right\}$. For large $P_{z}$, two poles $\left\{k_{4+}, \bar{k}_{4-}\right\}$ tend toward the origin, whereas the other two $\left\{k_{4-}, \bar{k}_{4+}\right\}$ tend toward infinity.

$k_{4+} \approx \frac{k_{\perp}^{2}+M_{1}^{2}-x^{2} m_{\pi}^{2}-i \epsilon}{2 x E} \quad \bar{k}_{4-} \approx \frac{k_{\perp}^{2}+M_{2}^{2}-\bar{x}^{2} m_{\pi}^{2}-i \epsilon}{-2 \bar{x} E}$ $k_{4-} \approx-2 x E+i \epsilon \quad \bar{k}_{4+} \approx 2 \bar{x} E-i \epsilon$

Notice that only for the physical domain $x \bar{x}>0$ do the poles close to the origin appear on each half-plane. In the unphysical domain $x \bar{x}<0$, both of these poles lie in the same half-plane, which after closing the contour in the opposite half-plane gives a vanishing result. We encapsulate this fact, that the leading-order PDAs have support only in the physical domain $x \bar{x}>0$, with on overal $\theta(x \bar{x})$. We close the contour for (A1) in the UHP, picking up only $\bar{k}_{4-}$ and $k_{4-}$. At the location of the first pole $\bar{k}_{4-}$, we have

$$
\begin{aligned}
y_{2}^{2}+M_{2}^{2} & =0 \quad y_{1}^{2} \approx \frac{1}{\bar{x}}\left[k_{\perp}^{2}+M_{2}^{2}-x \bar{x} m_{\pi}^{2}\right]-M_{2}^{2} \\
\bar{k}_{4-}-\bar{k}_{4+} & =-2 \bar{x} E .
\end{aligned}
$$

At the second pole $k_{4-}$, we have

$y_{1}^{2}+M_{1}^{2}=0 \quad y_{2}^{2} \approx-4 E^{2} x \quad k_{4-}-k_{4+}=-2 x E$. 


\section{APPENDIX B: NORMALIZATION $\lambda$ VALUES}

TABLE I. Values of $\lambda$ which normalize the DAs. A,P, and T correspond to axial-vector (twist-2), pseudoscalar (twist-3), and pseudotensor (twist-3) DAs respectively. "original" corresponds to our main expressions for the DA, (23), (27), and (28). The modifications " $\bmod (1)$ " and " $\bmod (2)$ " are discussed in Appendix C.

\begin{tabular}{llcc}
\hline \hline & Original & $\bmod (1)$ & $\bmod (2)$ \\
\hline$\lambda_{\pi}^{A}$ & 4.918 & 4.906 & 5.839 \\
$\lambda_{K}^{A}$ & 5.944 & 6.125 & 6.877 \\
$\lambda_{\pi}^{P}$ & 1.631 & 1.633 & 1.906 \\
$\lambda_{K}^{P}$ & 1.556 & 1.595 & 1.846 \\
$\lambda_{\pi}^{T}$ & 1.5337 & 1.538 & 1.988 \\
$\lambda_{K}^{T}$ & 1.466 & 1.594 & 1.932 \\
\hline \hline
\end{tabular}

\section{APPENDIX C: MODIFICATIONS TO THE CONSTITUENT QUARK MASS}

Our effective quark mass obtained at leading order in $\alpha$ is given by (16). A plot of the zero-momentum limit as a function of current mass $m$ is shown in Fig. 2. This constituent quark mass has the interesting property of being approximately constant for $0 \mathrm{MeV}<m<180 \mathrm{MeV}$, assuming phenomenological values of $\rho$ and $\alpha$. Although obtained from a $O(\alpha)$ integral equation, (16) contains higher powers of $\alpha$ resummed into dependence on $m$ through $\xi$. If we maintain strict power counting in $\alpha$ and simultaneously assume sufficiently small quark mass $m$ such that $\xi \ll 1$ (for our parameter values this is not true), then we arrive at the approximation $M(k, m) \approx M(k)+m$.
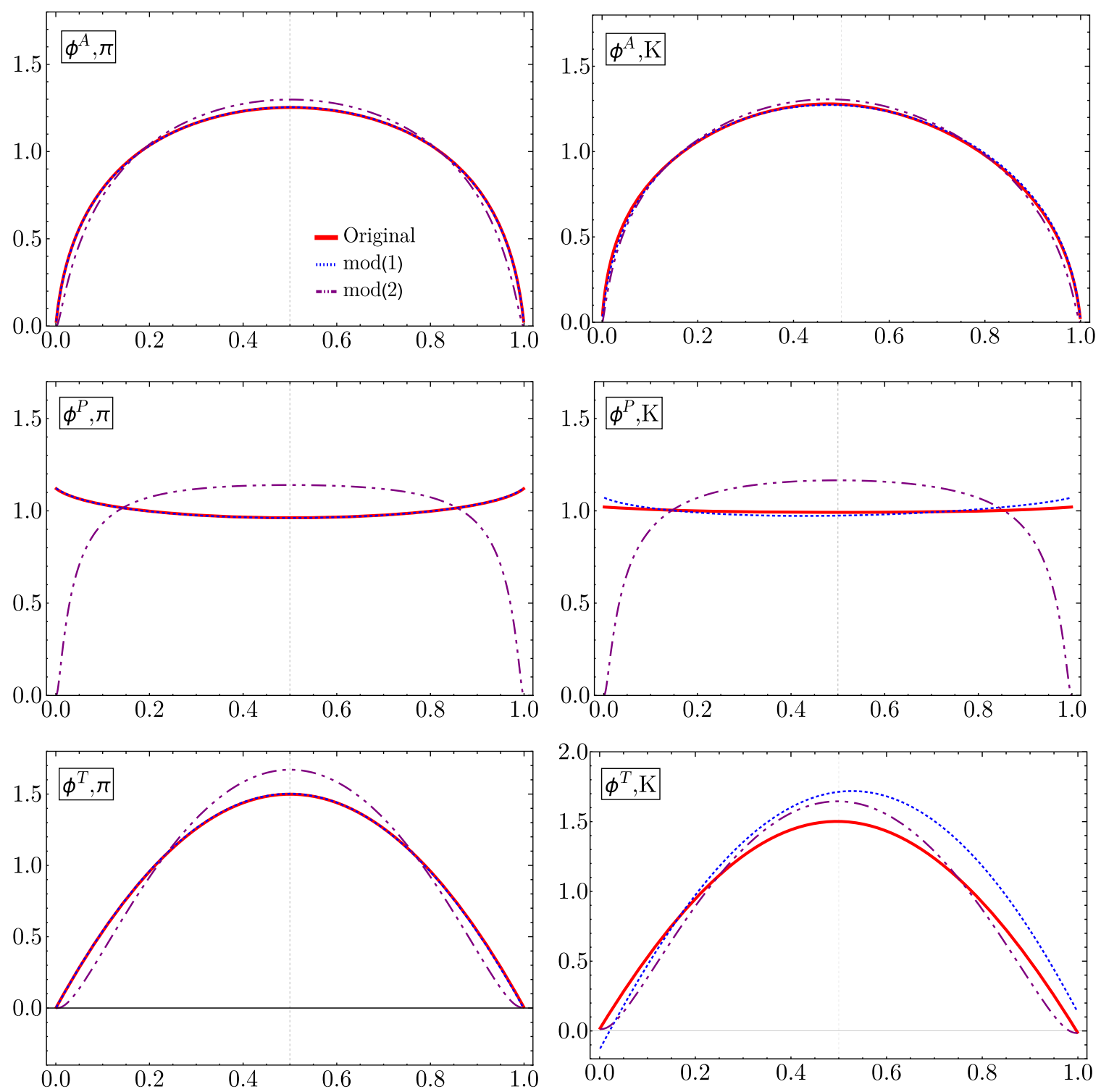

FIG. 6. Unevolved DA, at $Q=Q_{0}=631 \mathrm{MeV}$, with certain modifications to the effective mass. "Original" (red-thick-solid) denotes (23), (27), and (28). "mod(1)" (blue-dotted) denotes the shifted effective mass, and "mod(2)" (purple-dash-dot-dot) the shifted cutoff. See text. 
We insert this approximation into the expressions for the DAs and see how they change. In Fig. 6, this change is denoted by " $\bmod (1)$." The most notable resulting change is seen in the kaon pseudotensor DA, which no longer approaches zero at $x \rightarrow 1$. A slight restoration of $x \leftrightarrow \bar{x}$ symmetry is seen in the kaon twist-2 DA. All other DAs are relatively unchanged.
Finally, we consider the effect of restricting $k_{\perp}>M(0, m)$ in the cutoff (A3), as was noted in Sec. IV. This modification was implicitly present in our previous paper [17]. In Fig. 6 this change is denoted by " $\bmod (2) . "$ This results in a slight narrowing of the axial-vector (twist-2) and pseudotensor (twist-3) DAs. The biggest change is seen in the pseudoscalar (twist-3) DA, which becomes concave.
[1] X. Ji, Parton Physics on a Euclidean Lattice, Phys. Rev. Lett. 110, 262002 (2013).

[2] J.-H. Zhang, J.-W. Chen, X. Ji, L. Jin, and H.-W. Lin, Pion distribution amplitude from lattice QCD, Phys. Rev. D 95, 094514 (2017).

[3] X. Ji, A. Schäfer, X. Xiong, and J.-H. Zhang, One-loop matching for generalized parton distributions, Phys. Rev. D 92, 014039 (2015).

[4] G. S. Bali, V. M. Braun, B. Gläßle, M. Göckeler, M. Gruber, F. Hutzler, P. Korcyl, A. Schäfer, P. Wein, and J.-H. Zhang, Pion distribution amplitude from Euclidean correlation functions: Exploring universality and higher-twist effects, Phys. Rev. D 98, 094507 (2018).

[5] C. Alexandrou, K. Cichy, M. Constantinou, K. Jansen, A. Scapellato, and F. Steffens, Transversity parton distribution functions from lattice QCD, Phys. Rev. D 98, 091503 (2018).

[6] T. Izubuchi, L. Jin, C. Kallidonis, N. Karthik, S. Mukherjee, P. Petreczky, C. Shugert, and S. Syritsyn, Valence parton distribution function of pion from fine lattice, Phys. Rev. D 100, 034516 (2019).

[7] T. Izubuchi, X. Ji, L. Jin, I. W. Stewart, and Y. Zhao, Factorization theorem relating Euclidean and light-cone parton distributions, Phys. Rev. D 98, 056004 (2018).

[8] X. Ji, Y. Liu, and I. Zahed, Quasiparton distribution functions: Two-dimensional scalar and spinor QCD, Phys. Rev. D 99, 054008 (2019).

[9] R. A. Briceño, M. T. Hansen, and C. J. Monahan, Role of the euclidean signature in lattice calculations of quasidistributions and other nonlocal matrix elements, Phys. Rev. D 96, 014502 (2017).

[10] A. V. Radyushkin, Pion distribution amplitude and quasidistributions, Phys. Rev. D 95, 056020 (2017).

[11] Y.-Q. Ma and J.-W. Qiu, Extracting parton distribution functions from lattice QCD calculations, Phys. Rev. D 98, 074021 (2018).

[12] M. C. Chu, J. M. Grandy, S. Huang, and J. W. Negele, Evidence for the role of instantons in hadron structure from lattice QCD, Phys. Rev. D 49, 6039 (1994).

[13] D. Diakonov and V. Petrov, Confining ensemble of dyons, Phys. Rev. D 76, 056001 (2007).

[14] Y. Liu, E. Shuryak, and I. Zahed, Confining dyon-antidyon Coulomb liquid model. I., Phys. Rev. D 92, 085006 (2015).

[15] J. Greensite, Confinement from center vortices: A review of old and new results, EPJ Web Conf. 137, 01009 (2017).
[16] J. C. Biddle, W. Kamleh, and D. B. Leinweber, Visualization of center vortex structure, Phys. Rev. D 102, 034504 (2020).

[17] A. Kock, Y. Liu, and I. Zahed, Pion and kaon parton distributions in the QCD instanton vacuum, Phys. Rev. D 102, 014039 (2020).

[18] S.-I. Nam and H.-C. Kim, Twist-3 pion and kaon distribution amplitudes from the instanton vacuum with flavor SU(3) symmetry breaking, Phys. Rev. D 74, 096007 (2006).

[19] H.-M. Choi and C.-R. Ji, Twist-3 distribution amplitudes of pion in the light-front quark model, Few Body Syst. 58, 31 (2017).

[20] E. V. Shuryak, The role of instantons in quantum chromodynamics. 1. Physical vacuum, Nucl. Phys. B203, 93 (1982).

[21] T. Schfer and E. V. Shuryak, Instantons in QCD, Rev. Mod. Phys. 70, 323 (1998).

[22] P. J. Moran and D. B. Leinweber, Buried treasure in the sand of the QCD vacuum, in QCD Downunder II (2008), arXiv:0805.4246.

[23] X. Ji, Y. Liu, Yu.-S. Liu, J.-H. Zhang, and Y. Zhao, Largemomentum effective theory, Rev. Mod. Phys. 93, 035005 (2021).

[24] Y. Liu and I. Zahed, Small size instanton contributions to the quark quasi-PDF and matching kernel, arXiv:2102 .07248 .

[25] A. Hasenfratz, Spatial correlation of the topological charge in pure SU(3) gauge theory and in QCD, Phys. Lett. B 476, 188 (2000).

[26] E. V. Shuryak, Probing the boundary of the nonperturbative QCD by small size instantons, arXiv:hep-ph/9909458.

[27] B. V. Geshkenbein and M. V. Terentev, The enhanced power correction to the asymptotics of the pion form-factor, Phys. Lett. 117B, 243 (1982).

[28] E. Shuryak and I. Zahed, Nonperturbative quark-antiquark interactions in mesonic form factors, Phys. Rev. D 103, 054028 (2021).

[29] P. O. Bowman, U. M. Heller, D. B. Leinweber, A. G. Williams, and J. Zhang, Infrared and ultraviolet properties of the landau gauge quark propagator, Nucl. Phys. B, Proc. Suppl. 128, 23 (2004), proceedings of the 2nd Cairns Topical Workshop on Lattice Hadron Physics.

[30] W. Broniowski and E. R. Arriola, Nonperturbative partonic quasidistributions of the pion from chiral quark models, Phys. Lett. B 773, 385 (2017). 
[31] S. Jia and J. P. Vary, Basis light front quantization for the charged light mesons with color singlet Nambu-JonaLasinio interactions, Phys. Rev. C 99, 035206 (2019).

[32] M. Ding, K. Raya, D. Binosi, L. Chang, C. D. Roberts, and S. M. Schmidt, Symmetry, symmetry breaking, and pion parton distributions, Phys. Rev. D 101, 054014 (2020).

[33] M. Shifman and M. Vysotsky, Form factors of heavy mesons in QCD, Nucl. Phys. 186, 475 (1981).

[34] R. Zhang, C. Honkala, H.-W. Lin, and J.-W. Chen, Pion and kaon distribution amplitudes in the continuum limit, Phys. Rev. D 102, 094519 (2020).
[35] J. P. B. C. de Melo, I. Ahmed, and K. Tsushima, Parton distribution in pseudoscalar mesons with a light-front constituent quark model, AIP Conf. Proc. 1735, 080012 (2016).

[36] E. M. Aitala et al. (Fermilab E791 Collaboration), Direct Measurement of the Pion Valence-Quark Momentum Distribution, the Pion Light-Cone Wave Function Squared, Phys. Rev. Lett. 86, 4768 (2001).

[37] C. Shi, C. Chen, L. Chang, C. D. Roberts, S. M. Schmidt, and H.-S. Zong, Kaon and pion parton distribution amplitudes to twist three, Phys. Rev. D 92, 014035 (2015).

[38] I. Zahed, Mass sum rule of hadrons in the QCD instanton vacuum, Phys. Rev. D 104, 054031 (2021). 